\title{
Predicting visual acuity in Bietti crystalline dystrophy: evaluation of image parameters
}

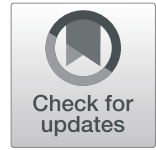

Chu-Yen Huang 1,2, Eugene Yu-Chuan Kang ${ }^{1,2}$, Lung-Kun Yeh ${ }^{1,2}$, An-Lun Wu ${ }^{3}$, Pei-Kang Liu 4,5,6,7, I-Wen Huang ${ }^{4}$, Joseph Ryu ${ }^{4}$, Laura Liu ${ }^{1,2}$, Wei-Chi Wu ${ }^{1,2}$, Chi-Chun Lai ${ }^{1,2,8}$, Kuan-Jen Chen ${ }^{1,2^{*}}$ and Nan-Kai Wang ${ }^{4^{*}}$ (D)

\begin{abstract}
Background: To analyze multiple imaging modalities in patients with Bietti crystalline dystrophy (BCD) and to investigate which factors from these modalities are associated with best corrected visual acuity (BCVA).

Methods: In this retrospective study, 40 eyes from 22 patients with BCD were included and were separated into group 1 (BCVA $\leq 20 / 200)$ and group 2 (BCVA > 20/200). Data including BCVA and characteristic findings from nearinfrared reflectance (NIR) imaging, fundus autofluorescence (FAF), and spectral domain-optic coherence tomography (SD-OCT) were analyzed and compared. The outcome measures of multimodal imaging were evaluated for correlation with BCVA.

Results: NIR is a good diagnostic tool for detecting either crystalline or sclerotic vessels in BCD. Patients in group 1 tended to have a thinner choroid $(P=0.047)$ with ellipsoid zone (EZ) disruption $(P=0.011)$. Calculation of the area under the curve indicated that EZ disruption detected on SD-OCT could be a good predictor of legal blindness in $\mathrm{BCD}$.

Conclusion: For the diagnosis of BCD, NIR could be a good diagnostic tool. Of the studied imaging modalities, we found that EZ disruption at the fovea were strongly associated with legal blindness, which could be easily assessed by SD-OCT.
\end{abstract}

Keywords: Best corrected visual acuity (BCVA), Bietti crystalline dystrophy (BCD), Choroidal thickness, Crystalline, Ellipsoid zone disruption, Fundus autofluorescence (FAF), Near-infrared reflectance (NIR), Sclerotic vessels, Spectral domain-optical coherence tomography (SD-OCT)

\section{Background}

Bietti crystalline dystrophy (BCD) (OMIM\#210370) is a severe inherited retinal dystrophy that is caused by autosomal recessive mutations in the cytochrome P450 family 4 subfamily $V$ member 2 (CYP4V2) gene [1]. The CYP4V2 enzyme is involved in fatty acid oxidation, and the protein is highly expressed in retinal pigment epithelium (RPE) cells. As such, it is postulated that CYP4V2

\footnotetext{
* Correspondence: cgr999chiayi@yahoo.com.tw; wang.nankai@gmail.com 'Department of Ophthalmology, Chang Gung Memorial Hospital, Linkou Medical Center, Taoyuan, Taiwan

${ }^{4}$ Department of Ophthalmology, Edward S. Harkness Eye Institute, Columbia University Irving Medical Center, Columbia University, New York, USA

Full list of author information is available at the end of the article
}

defects affect lipid breakdown [2]; however, the precise pathology of how these mutations lead to photoreceptor degeneration remains unknown. Given our limited understanding of disease pathogenesis, there is currently no available treatment for BCD.

The disease was first described by Bietti in 1937 based on three patients who exhibited numerous crystal deposits in the retina and perilimbal cornea. Additional clinical characteristics include nyctalopia and visual field defects that manifest in the second to fourth decade of life [3]. As the disease progresses, crystal deposits gradually decrease in number [4], and retinal and choroid atrophy develop together with progressive visual loss [5].

(c) The Author(s). 2021 Open Access This article is licensed under a Creative Commons Attribution 4.0 International License, which permits use, sharing, adaptation, distribution and reproduction in any medium or format, as long as you give appropriate credit to the original author(s) and the source, provide a link to the Creative Commons licence, and indicate if changes were made. The images or other third party material in this article are included in the article's Creative Commons licence, unless indicated otherwise in a credit line to the material. If material is not included in the article's Creative Commons licence and your intended use is not permitted by statutory regulation or exceeds the permitted use, you will need to obtain permission directly from the copyright holder. To view a copy of this licence, visit http://creativecommons.org/licenses/by/4.0/ The Creative Commons Public Domain Dedication waiver (http://creativecommons.org/publicdomain/zero/1.0/) applies to the data made available in this article, unless otherwise stated in a credit line to the data. 
To date, several studies have characterized the clinical features of BCD using fundus photography [6], fluorescence angiography, fundus autofluorescence (FAF), spectral domain-optical coherence tomography (SD-OCT) and near-infrared reflectance (NIR) imaging [7]. Although fundus photography, FAF and SD-OCT have been used to classify different stages of $\mathrm{BCD}$ [8], no study has analyzed the correlation of these image characteristics with visual function. Recently, Miyata et al. reported a correlation between BCD features on OCT/ optical coherence tomography angiography (OCTA) and visual function including visual acuity (VA) and visual field (VF) [9]. However, it is unknown whether features observed in other multimodal imaging techniques such as color fundus, FAF and NIR could be used as predictors of visual function.

The purpose of this study is to compare the results of several retinal imaging modalities in BCD patients and identify indicators of visual function. Such indicators from noninvasive imaging modalities can provide a thorough assessment of retinal structure and progression of the disease. Several clinical trials of gene therapy for inherited retinal dystrophies have been initiated after the United State Food and Drug Administration approved the first RPE65 gene therapy [10]. Although there is currently no definite treatment for $\mathrm{BCD}$, reliable indicators that correlate with visual function and capture changing structural and functional features of BCD may serve as potential tools for monitoring disease progression as well as valuable outcome measures for future gene therapy in interventional trials.

\section{Methods}

\section{Study population}

We retrospectively reviewed patients with $\mathrm{BCD}$ who visited the Department of Ophthalmology at Chang Gung Memorial Hospital in Linkou Medical Center between January 2010 and December 2018. The study was approved by the Institutional Review Board of Chang Gung Memorial Hospital, Taiwan (IRB 202001456B0). BCD was clinically diagnosed based on patients' symptoms and retinal images. The characteristic features of $\mathrm{BCD}$ include the presence of crystalline deposits at the posterior pole, prominent sclerotic choroidal vessels, choroidretinal dystrophy, patchy atrophy of FAF, with or without outer retinal tubulation (ORT) on SD-OCT.

\section{Clinical examination}

All the patients underwent a complete ocular examination at their first visit, including measurement of BCVA, refractive error, intraocular pressure, dilated fundus examination, fundus photography, SD-OCT, NIR imaging and FAF imaging. Some patients also received fluorescence angiography, a static VF test, blue-light full-field electroretinogram, or genetic testing for CYP4V2 mutations. NIR and FAF were performed with a confocal scanning laser ophthalmoscope (Heidelberg Retina Angiograph HRA2; Heidelberg Engineering, Dossenheim, Germany). SD-OCT images were obtained using a Spectralis HRA-OCT system (Heidelberg Engineering). Patients with other ocular diseases that could also attribute to prominently decreased vision were excluded.

\section{Acquisition of Image Biometrics}

The results of the imaging modalities analyzed in our study were collected on the first visit for each patient. All of the characteristics of each imaging modality were classified by at least three independent investigators blinded to the associated BCVA. The presence of crystalline deposits and sclerotic vessels at the posterior pole was determined on either fundus photography or NIR. Foveal retinal thickness (average thickness at $2 \mathrm{~mm}$ diameter from the center of the fovea), subfoveal choroidal thickness, ellipsoid zone (EZ) line integrity at the fovea, and the presence of outer retinal tubulation (ORT) were identified from the SD-OCT images. The presence or absence of ORT was determined at the center of a horizontal and vertical $8.0 \mathrm{~mm}$ SD-OCT scan cross. The severity of changes in FAF images was graded as described by Li et al. in 2015 [8], based on the staging system established by Yuzawa et al. [6] Stage 1 was defined as normal AF or hyper/hypo-AF spots confined to the posterior pole. Stage 2 was defined as confluent hypo-AF patches extending beyond the posterior pole. Stage 3 was defined as the clear absence of AF at the posterior pole. Retinal and choroidal biometry was measured using the built-in software in the SD-OCT system (Heidelberg Engineering). Subfoveal choroidal thickness was defined as the distance between the outer borders of the RPE and the hyperrefractive line behind the large vessel layers of the choroid and was measured manually at the subfovea using build-in software by two independent investigators.

\section{Statistical analysis}

All the eyes were divided into two groups according to BCVA at first visit. Group 1 consists of patients with BCVA $\leq 20 / 200$, and group 2 includes patients with BCVA $>20 / 200$. BCVA $\leq 20 / 200$ was defined as legal blindness according to National Eye Institute of the US [11].

Statistical analysis was performed using SPSS (IBM SPSS Statistics for Windows, Version 21.0. IBM Corp. Armonk, NY). Continuous covariates were assessed using an independent $t$ test. A Mann-Whitney $U$ test was performed for those covariates that were not normally distributed. Categorical covariates were assessed using the Pearson chi-squared test, or with Fisher's exact 
test if the expected value was $<5$. Univariate regression was calculated for variations in BCVA relative to age, sex, crystalline deposits, central retinal thickness, subfoveal choroidal thickness, EZ disruption at the fovea, ORT, sclerotic vessels, and FAF staging. Multiple logistic regression was applied to evaluate the explanatory variables with regard to the dependent variable, including age, subfoveal choroidal thickness and EZ disruption at the fovea. The cutoff values of these biometrics that predicted BCVA $\leq 20 / 200$ were analyzed using a receiveroperating characteristic (ROC) curve. The accuracy was measured by the area under the curve (AUC). An AUC of 1.0 represents a perfect test for discrimination, whereas an AUC of 0.5 indicates random assignment. Statistical significance was defined as a $P$-value $<0.05$.

\section{Results}

\section{Patient demographics}

In total, 40 eyes from 22 patients with $\mathrm{BCD}$ were included and analyzed in our study. Patients with choroidal neovascularization ( 2 eyes), glaucoma ( 1 eye), and macula scar (1 eye) were excluded. Among these 22 patients clinically diagnosed with $\mathrm{BCD}$, four had previously undergone genetic testing from a prospective study (Genetic study in hereditary retinal and optic nerve diseases, IRB 201601569B0C601), all of whom were confirmed to have the homozygous mutation c.802-8 810del17insGC in CYP4V2 gene (Fig. 1), which is the most common pathogenic variant of $\mathrm{BCD}$ in Eastern countries $[12,13]$. Of the 40 eyes, 14 had a BCVA $\leq 20$ / 200 and were classified as group 1 (35\%), while 26 had a BCVA $>20 / 200$ and were classified as group $2(65 \%)$. The mean age of the participants was $46.80 \pm 12.2$ years (mean $\pm \mathrm{SD}$ ). The mean follow-up period was $30.7 \pm$ 28.39 months (mean $\pm \mathrm{SD}$ ).

\section{Features in imaging modalities}

NIR can easily identify crystalline deposits and sclerotic vessels (Figs. 2, 3a, b, 4a, b). The crystalline deposits decreased in number as the disease progressed (Fig. 2a, b) and nearly disappeared in advanced stages of disease (Fig. 2d). By contrast, sclerotic choroidal vessels were absent during the early stages of disease (Fig. 2a, b) but became more prominent later (Fig. 2c, d). In our patients, all five eyes without crystalline deposits on their fundus had prominent sclerotic choroid vessels on their NIR images, which indicated later stages of BCD.

Using SD-OCT, disruption of the EZ can be observed in the affected area (Fig. 3c). These lesions initially presented as random patches with disruption of the EZ, followed by abrupt outer nuclear layer destruction. The retinal layers near the fovea (Fig. 3c, e) were usually preserved on SD-OCT until later stages of disease. Figure 4 shows examples of a patient with bilateral symmetric amounts of crystalline deposit and sclerotic vessels on color fundus (Fig. 4a) and NIR (Fig. 4b), similar FAF stages (Fig. 4c), and similar subfoveal choroidal thickness in both eyes. However, one eye had a preserved EZ at the fovea while the other eye did not. The BCVA of the eye with a disrupted EZ at the fovea was 20/1000, which is considered to be legally blind, whereas BCVA of the other eye with a preserved island at the fovea was 20/50.

FAF in BCD initially presented with some hyper- and hypo-AF spots. These hypo-AF spots gradually enlarged in size to hypo-AF patches and eventually became confluent (Fig. 5). A hypo-AF signal indicates RPE atrophy or RPE death and usually appears together with a disrupted EZ on SD-OCT, indicating photoreceptor degeneration. However, in some cases, we found that the area of disrupted EZ on SD-OCT did not necessarily correspond to the hypo-AF patches (Fig. 4c, white stars).

\section{Comparison of the two groups}

The comparison of the two study groups is summarized in Table 1. The mean age of group 1 was $52.21 \pm 13.47$ years and that of group 2 was $43.88 \pm 10.60$ years $(P=0.038$, independent $\mathrm{t}$ test). Refractive errors were $-2.26 \mathrm{D} \pm 2.65 \mathrm{D}$ in group 1 , and $-3.72 \mathrm{D} \pm 3.78 \mathrm{D}$ in group $2(P=0.589$, Mann-Whitney U test). 25 eyes in group 2 (96\%) had crystalline deposits on the fundus, but crystals could only be observed in 10 eyes in group $1(71 \%)(P=0.043$, Fisher's exact test). The mean central retinal thickness was $256.86 \mu \mathrm{m} \pm 39.40 \mu \mathrm{m}$ in group 1 and $272.73 \mu \mathrm{m} \pm$ $57.86 \mu \mathrm{m}$ in group $2(P=0.366$, independent $\mathrm{t}$ test). The mean subfoveal choroidal thickness was $73.93 \mu \mathrm{m} \pm$ $43.31 \mu \mathrm{m}$ in group 1 and $126.20 \mu \mathrm{m} \pm 50.02 \mu \mathrm{m}$ in group 2 ( $P=0.002$, independent $t$ test). 13 eyes in group 1 (92.9\%) and 5 eyes in group $2(19.2 \%)$ showed EZ disruption at the fovea $(P<0.001$, Fisher's exact test).

Regarding the presence of ORT, 5 eyes in group 1 (35\%) and 15 eyes in group $2(57 \%)$ had ORT that was detected in either horizontal and/or vertical 8.0-mm scanning at the center SD-OCT $(P=0.185)$. Sclerotic vessels were observed on NIR images in 13 eyes in group 1 (93\%) and 22 eyes in group 2 (85\%) ( $P=0.137$, Pearson Chi-square). FAF images were classified into three stages. In group 1 , only 1 eye (7\%) was classified as stage 1, 5 eyes were classified as stage $2(36 \%)$, and 7 eyes were classified as stage 3 (50\%). In group 2, 4 eyes were classified as stage $1(15 \%)$, 12 eyes were classified as stage $2(46 \%)$, and 4 eyes were classified as stage $3(15 \%)(P=0.122$, Pearson Chi-square).

\section{Statistics}

The univariate logistic regression analysis for legal blindness is shown in Table 2. Parameters including sex, refractive errors, central retinal thickness, presence of ORT, sclerotic vessels and FAF staging were not correlated significantly with legal blindness. On the other 

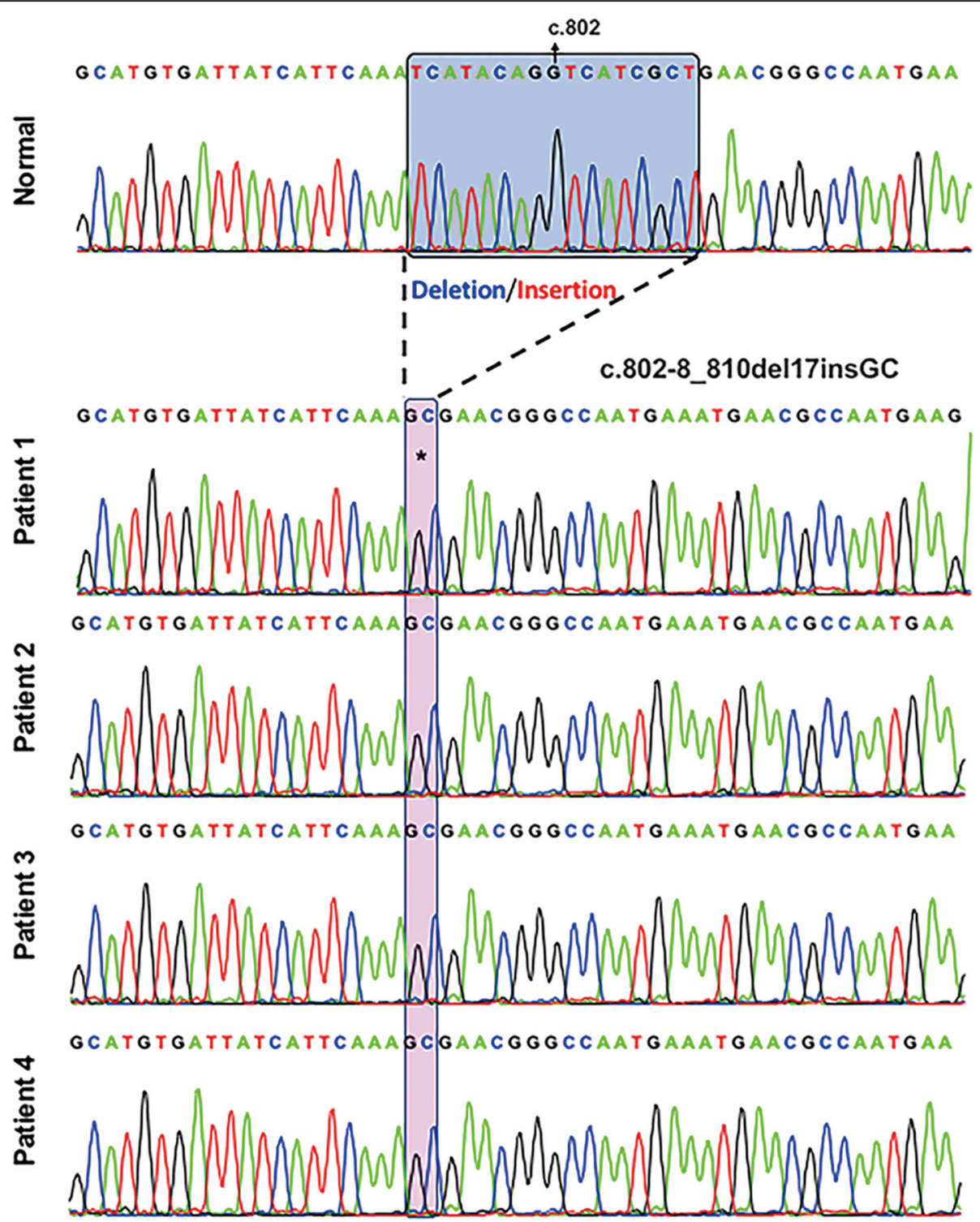

Fig. 1 Genetic analysis. 4 patients in our study received genetic analysis, and all of them had homozygous mutation c.802-8_810del17insGC in CYP4V2 gene. The blue box indicates the deletion of 17 nucleotides from intron position 802-8 to exon position 810 (coding sequence), and the red box showed that GC was inserted instead

hand, older age, decreased subfoveal choroidal thickness, EZ disruption at the fovea, and FAF stage 3 were correlated significantly with legal blindness.

We performed a stepwise multivariate regression to determine the explanatory variables most strongly associated with legal blindness, including subfoveal choroidal thinning $(P=0.047)$ and EZ disruption at the fovea $(P=0.011)$ (Table 2). The image biometric that most sensitively predicted BCVA $\leq 20 / 200$ in our study was EZ disruption at the fovea [Area under the curve (AUC): $0.868,95 \%$ confidence interval (CI): 0.747-0.989]. The ROCs for EZ disruption at the fovea, and subfoveal choroidal thickness as predictors of BCVA $\leq 20 / 200$ are shown in Fig. 6 . We also analyzed the data using a continuous variable for BCVA (converted to logMAR for statistic analysis) instead of dividing the patients into two groups. The results were similar in that age, subfoveal choroidal thickness, EZ disruption at the fovea, crystalline deposits, and FAF stage 3 were significantly related to BCVA (supplementary file).

\section{Discussion}

In this study, NIR was regarded as a convenient and noninvasive tool for the diagnosis of $\mathrm{BCD}$ because of its ability to detect crystalline deposits and sclerotic vessels in early and advanced stages of disease, respectively. We further explored the relationship between the features of 


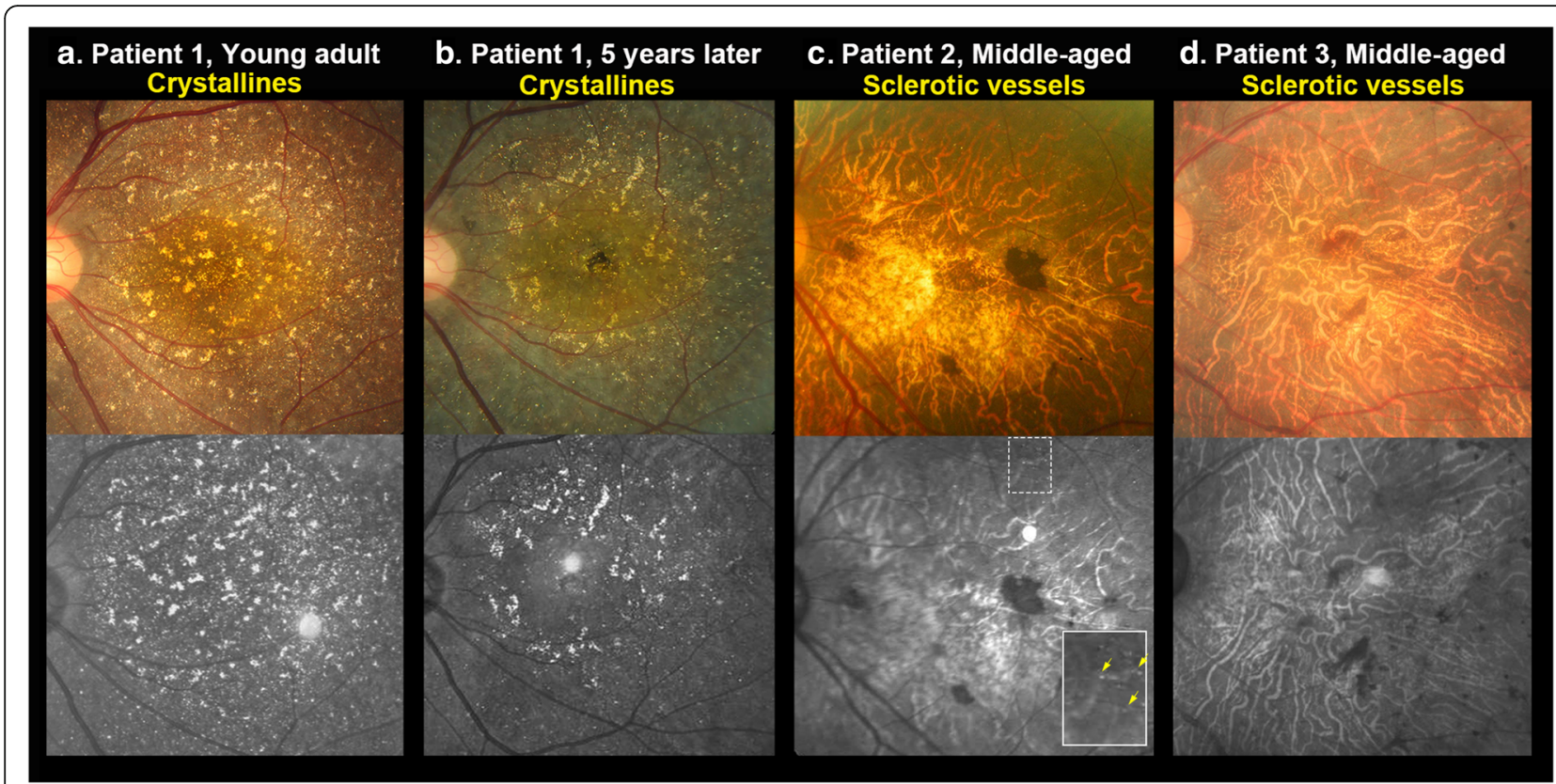

Fig. 2 NIR imaging. a Prominent hyperreflective crystalline deposits without sclerotic choroid vessels. $\mathbf{b}$ The color fundus and NIR images of the same patient after 5 years showing decreased crystalline deposits. There are still no sclerotic vessels on her NIR image. c Prominent sclerotic vessels have appeared. There are much fewer crystalline deposits. Some deposits are shown in the white dotted square (yellow arrows). $\mathbf{d}$ NIR image of advanced-stage BCD showing prominent sclerotic choroidal vessels and no crystalline deposits

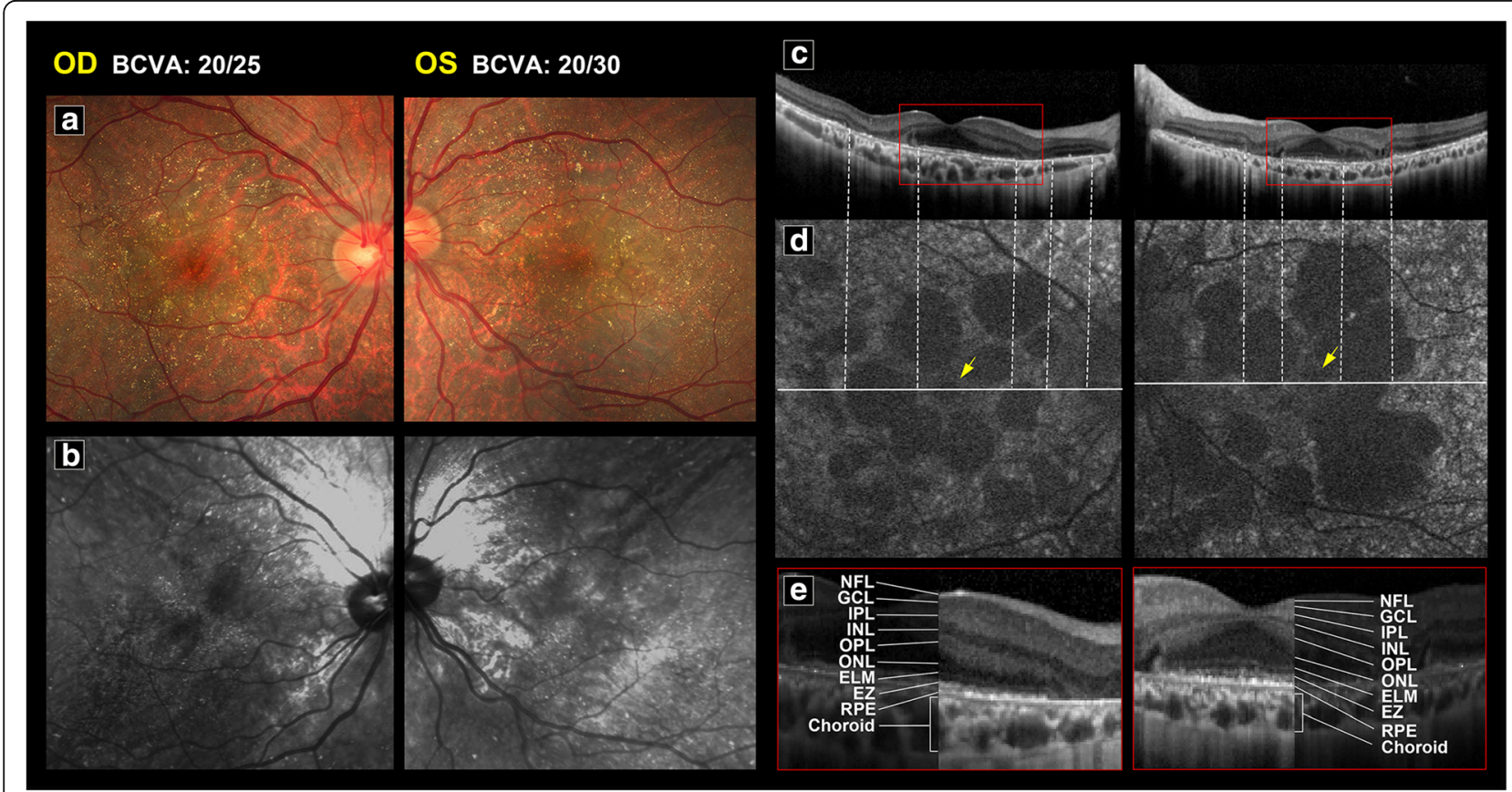

Fig. 3 An young adult with a diagnosis of BCD, BCVA of 20/22 (right eye, Oculus Dexter (OD)) and 20/29 (left eye, Oculus Sinister (OS)). a Color fundus showing obvious crystalline deposits. b Crystalline deposits can be clearly seen on NIR imaging. c OCT revealed preserved retinal structure near the fovea (red square) in both eyes. Outside the preserved island, outer retinal layer (ONL) destruction and EZ loss were observed. $\mathbf{d}$ In this case, some hypo-AF patches on FAF imaging corresponded to ONL and EZ destruction seen on OCT. Relatively low AF at the fovea (yellow arrows) corresponded to normal structures seen on OCT. e Enlarging the central island on SD-OCT, there are intact retinal structures in the central area (I NFL nerve fiber layer; GCL ganglion cell layer; IPL inner plexiform layer; INL inner nulear layer; OPL outer plexiform layer; ONL outer nuclear layer; ELM external limiting membrane; EZ ellipsoid zone; RPE retinal pigment epithelium) 


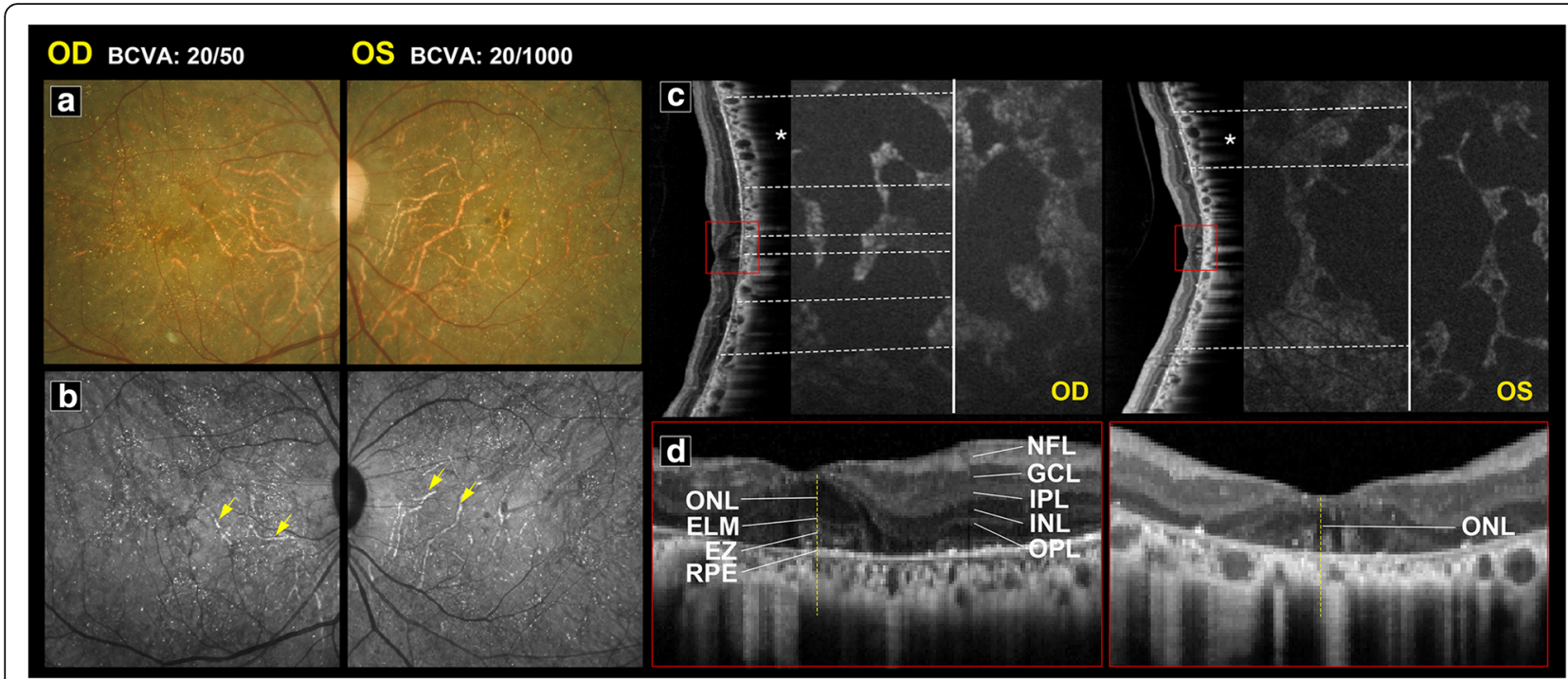

Fig. 4 This middle-aged patient had BCVA of 20/50 (OD) and 20/1000 (OS). a Color fundus revealed a greenish background color with depigmentation. b Obvious sclerotic vessels were evident on NIR (yellow arrows). Multiple hyperreflective crystal deposits could still be observed. c On $\mathrm{OCT}$, a small foveal preservation island was demonstrated in the right eye. OCT of the left eye showed destruction of EZ and ONL. A normal/hyper-AF area on AF cannot predict structural preservation on OCT. The white star indicates the area of outer nuclear layer thinning and EZ disruption seen on $\mathrm{OCT}$, but it corresponded to neither hypo-AF nor normal AF. $\mathbf{d}$ The foveal structures are labeled in high magnification from red squares in (c) (NFL nerve fiber layer; GCL ganglion cell layer; IPL inner plexiform layer; INL inner nulear layer; OPL outer plexiform layer; ONL outer nuclear layer; ELM external limiting membrane; EZ ellipsoid zone; RPE retinal pigment epithelium)

multimodal retinal imaging with legal blindness $(B C V A \leq 20 / 200)$. Notably, we found that EZ disruption at the fovea and decreased foveal choroidal thickness significantly correlated with legal blindness, while central retinal thickness, sclerotic vessels and FAF staging did not.

\section{NIR imaging}

The most characteristic feature of $\mathrm{BCD}$ is the presence of retinal crystalline deposits first described by Dr. Bietti in 1937 [3]. As the disease progresses, the crystals diminish and disappear because of RPE atrophy and

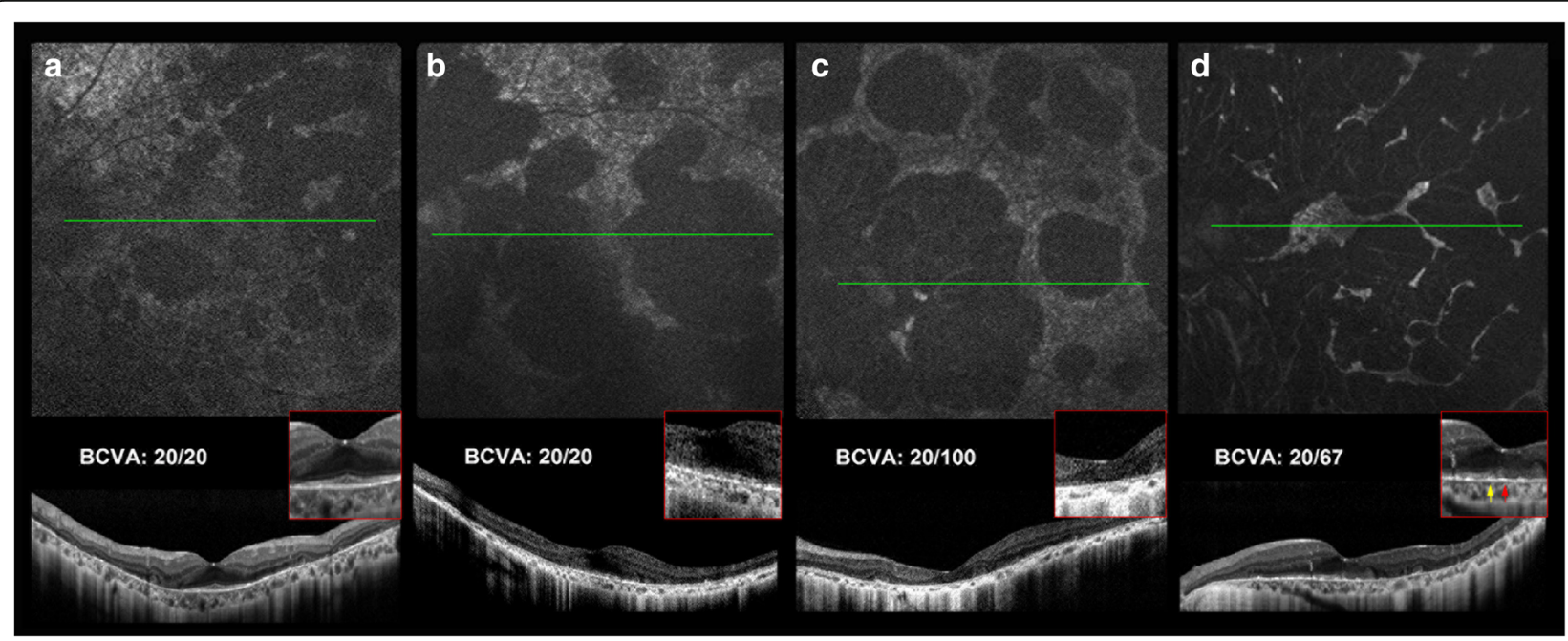

Fig. $\mathbf{5}$ It is hard to recognize a central island on FAF because of the hypo-AF at the fovea. $\mathbf{a}, \mathbf{b}$ The patients with preserved foveal structures, especially EZ, on OCT had a BCVA of 20/20. c OCT shows central destruction of the EZ. This eye had a BCVA of 20/100. On FAF, it was relatively difficult to determine whether the central area was preserved. $\mathbf{d}$ The preserved island of the normal retinal structure was eccentric. On OCT, a red arrow indicates the central fovea, but the EZ starts from the site where the yellowish arrow indicates the nasal retina. FAF shows confluent patches. The BCVA of this eye was 20/67 (FAF fundus autofluorescence; OCT optical choherence tomography; BCVA best corrected visual acuity; EZ ellipsoid zone) 
Table 1 Summary of clinical and demographic characteristics of patients in Group 1 and Group 2

\begin{tabular}{|c|c|c|c|c|c|c|c|}
\hline \multirow[t]{2}{*}{ Variables } & \multicolumn{2}{|c|}{ All patients } & \multicolumn{2}{|c|}{$\begin{array}{l}\text { Group } 1 \\
(B C V A \leq 20 / 200)(N=14)\end{array}$} & \multicolumn{2}{|c|}{$\begin{array}{l}\text { Group } 2 \\
(B C V A>20 / 200)(N=26)\end{array}$} & \multirow[t]{2}{*}{$p$-value } \\
\hline & Mean & SD & Mean & SD & Mean & SD & \\
\hline Age & 46.80 & 12.20 & 52.21 & 13.47 & 43.88 & 10.60 & ${ }^{*} 0.038$ \\
\hline Follow-up (months) & 30.70 & 28.39 & 28.27 & 27.99 & 35.21 & 29.61 & 0.747 \\
\hline Refractive error(D) & -3.29 & 3.51 & -2.26 & 2.65 & -3.72 & 3.78 & 0.589 \\
\hline Central retinal thickness $(\mu \mathrm{m})$ & 267.18 & 52.18 & 256.86 & 39.40 & 272.73 & 57.86 & 0.366 \\
\hline \multirow[t]{2}{*}{ Subfoveal Choroidal thickness $(\mu \mathrm{m})$} & 107.91 & 53.54 & 73.93 & 43.31 & 126.20 & 50.02 & ${ }^{*} 0.002$ \\
\hline & $\mathrm{N}$ & $\%$ & $N$ & $\%$ & $\mathrm{~N}$ & $\%$ & \\
\hline Male & 15 & 37.50 & 4 & 28.60 & 11 & 42.30 & 0.502 \\
\hline Crystalline deposits & 35 & 87.50 & 10 & 71.40 & 25 & 96.20 & ${ }^{*} 0.043$ \\
\hline EZ disruption at fovea & 18 & 45.00 & 13 & 92.90 & 5 & 19.20 & $*<0.001$ \\
\hline Outer retinal tubulation & 20 & 50.00 & 5 & 35.70 & 15 & 57.70 & 0.185 \\
\hline Sclerotic vessels & 35 & 87.50 & 13 & 92.90 & 22 & 84.60 & 0.640 \\
\hline FAF staging & & & & & & & 0.122 \\
\hline Stage 1 & 5 & 12.50 & 1 & 7.10 & 4 & 15.40 & 0.64 \\
\hline Stage 2 & 17 & 42.50 & 5 & 35.70 & 12 & 46.20 & 0.524 \\
\hline Stage 3 & 11 & 27.50 & 7 & 50.00 & 4 & 15.40 & ${ }^{*} 0.029$ \\
\hline
\end{tabular}

$B C V A$ best corrected visual acuity, $S D$ standard deviation, $N$ number, $E Z$ ellipsoid zone, $F A F$ fundus autofluorescence ${ }^{*} p<0.05$

thinning of the Bruch's membrane layer [14]. Pathologic analysis of crystalline deposits in BCD reported in 1987 revealed inclusion bodies in fibroblasts with lipid content within the crystalline deposits [15]. Oishi et al. reported that the presence of hyperreflective crystals on NIR has $100 \%$ sensitivity and $100 \%$ specificity for CYP4V2 mutations among patients with chorioretinal dystrophy [7]. However, crystalline deposits were barely detectable in five legally blind eyes (group 1). Because the crystalline deposits decrease as the disease progresses, the absence of crystalline deposits on fundus photography could indicate more severe or advanced stages of $\mathrm{BCD}$, which is usually accompanied by worse vision.

In addition to crystalline deposits, Dr. Bietti described "sclerotic vessels" in his three cases in 1937. ${ }^{1}$ The term

Table 2 Univariate and multivariate analysis of BCVA for Group 1 and Group 2

\begin{tabular}{|c|c|c|c|c|c|c|c|c|}
\hline \multirow{3}{*}{$\frac{\text { Variables }}{\text { Age }}$} & \multicolumn{4}{|c|}{ Univariate analysis } & \multicolumn{4}{|c|}{ Multivariate analysis } \\
\hline & \multirow{2}{*}{$\begin{array}{l}\text { OR } \\
1.064\end{array}$} & \multicolumn{2}{|c|}{$95 \% \mathrm{Cl}$} & \multirow{2}{*}{$\begin{array}{l}p \text {-value } \\
* 0.047\end{array}$} & \multirow{2}{*}{$\begin{array}{l}\text { OR } \\
0.751\end{array}$} & \multicolumn{2}{|c|}{$95 \% \mathrm{Cl}$} & \multirow{2}{*}{$\frac{\boldsymbol{p} \text {-value }}{0.069}$} \\
\hline & & 1.001 & 1.131 & & & 0.551 & 1.023 & \\
\hline Follow-up (months) & 1.009 & 0.986 & 1.032 & 0.457 & & & & \\
\hline Refractive error(D) & 1.149 & 0.896 & 1.473 & 0.275 & & & & \\
\hline Central retinal thickness $(\mu \mathrm{m})$ & 0.994 & 0.98 & 1.007 & 0.359 & & & & \\
\hline Subfoveal Choroidal thickness ( $\mu \mathrm{m})$ & 0.975 & 0.957 & 0.993 & $* 0.007$ & 0.904 & 0.819 & 0.999 & $* 0.047$ \\
\hline Male & 0.545 & 0.135 & 2.204 & 0.395 & & & & \\
\hline Crystalline deposits & 10 & 0.992 & 100.921 & 0.051 & & & & \\
\hline EZ disruption at fovea & 0.018 & 0.002 & 0.175 & ${ }^{*}<0.001$ & 0.002 & 0 & 0.234 & $* 0.011$ \\
\hline Outer retinal tubulation & 2.455 & 0.642 & 9.391 & 0.19 & & & & \\
\hline Sclerotic vessels & 0.423 & 0.043 & 4.203 & 0.463 & & & & \\
\hline \multicolumn{9}{|l|}{ FAF staging } \\
\hline Stage 1 & 0.423 & 0.043 & 4.203 & 0.463 & & & & \\
\hline Stage 2 & 1.543 & 0.405 & 5.879 & 0.525 & & & & \\
\hline Stage 3 & 5.5 & 1.234 & 24.506 & $* 0.025$ & 0.076 & 0.003 & 2.069 & 0.126 \\
\hline
\end{tabular}

$B C V A$ best corrected visual acuity, $O R$ odds ratio, $C$ confidence interval, Sig. significance; $E Z$ ellipsoid zone, $F A F$ fundus autofluorescence ${ }^{*} p<0.05$ 


\section{ROC curve}

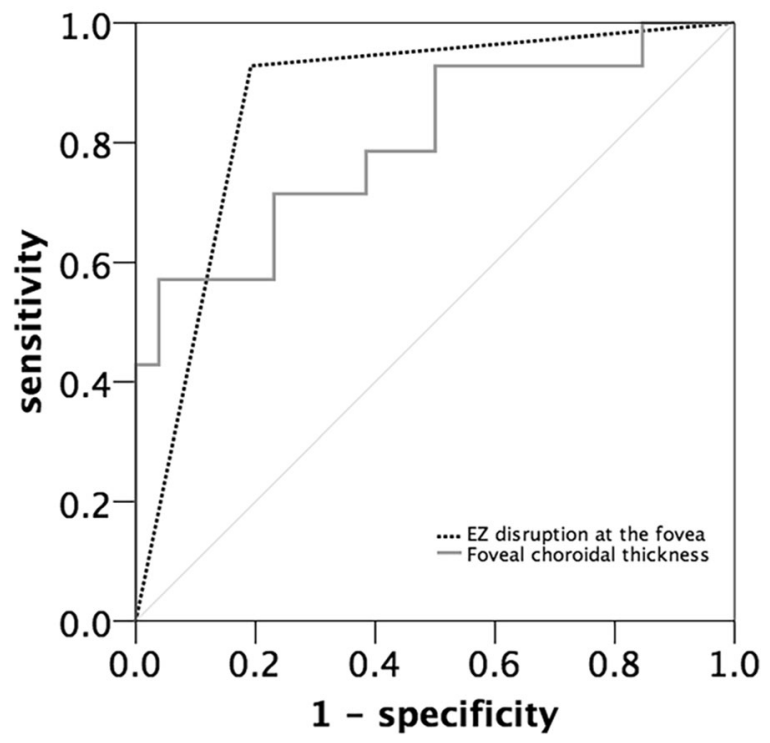

Fig. 6 Receiver-operating characteristic (ROC) for two factors. "EZ disruption at fovea" (the dot line) showed the largest area under the curve (AUC) of 0.868 , indicating the best discriminatory power. The AUCs of foveal choroidal thickness (the grey line) was 0.802 (EZ ellipsoid zone)

sclerotic choroidal vessels refers to fibrotic, hyalinization, and occlusion of choroidal vessels. We found that large choroidal vessels in BCD are hyperreflective in NIR (Fig. 2). Kaiser-Kupfer et al. reported the thinned and diffuse fibrotic choroid with mixed with hypertrophic and hyperplastic melanocytes in pathology [16]. It is interesting to note that some retinal lesions such as lacquer cracks also presented as "hyperrefective" in NIR, which are associated with fibrotic tissue after the Bruch's membrane ruptures in highly myopic eyes [17]. In our patients, all five legally blind eyes without obvious crystalline deposits had prominent sclerotic vessels on NIR. The large hyperreflective choroidal vessels on NIR are uncommon in other retinal degenerative conditions such as choroideremia or non-syndromic retinitis pigmentosa. Therefore, NIR imaging can be a useful tool to diagnose BCD as it is able to detect not only crystalline deposits in the early stages of disease but also sclerotic choroidal vessels in more advanced stages when crystalline deposits are invisible (Fig. 2).

\section{SD-OCT imaging}

Several patients in our study had asymmetric BCVA between their two eyes even though image modalities showed similar crystalline deposits, similar FAF stage and pattern, prominent sclerotic vessels on NIR, and similar central retinal thickness. Notably, SD-OCT in these patients showed preserved central islands in the eye with better vision and destruction of the normal retinal lamella at the fovea in the legally blind eye (Fig. 4). The results of our statistical analysis revealed that EZ disruption at the fovea and thinner subfoveal choroid were strong indicators of legal blindness (Fig. 6). Central ellipsoid-preserved island could also be recognized on En face optical coherence tomography [18]. Miyata et al. used features of SD-OCT and OCTA to demonstrate a correlation between decreased BCVA and progression of VF defects [9]. Their results showed that a remaining central choroid capillary seen on OCTA, as well as foveal EZ disruption and choroid thickness seen on OCT, were well correlated with BCVA and VF. In this study, we analyzed the correlation of BCVA with the characteristics of other image modalities. Although fundus photo, NIR, and FAF can be used to monitor different stages of $\mathrm{BCD}[6,8]$, we found that the characteristics on SDOCT are still the strongest predictor for BCVA in BCD.

ORT was first described on SD-OCT in age related macular degeneration; however, it can be observed in other degenerative retinal disorders [19]. It has been suggested that ORT follows photoreceptor loss or retinal injuries in several retinal degeneration. However, it is not a unique feature of BCD. Because ORT structures were located outside the foveola on cross-sectional SDOCT in BCD, their presence or absence is not related to BCVA in our study.

\section{FAF and SD-OCT correlation}

Halfort et al. correlated FAF images with SD-OCT images in BCD patients, and found that a well-defined hypo-AF edge corresponded to abrupt outer retinal atrophy seen on SD-OCT [20]. However, in our study, we found that outer retinal atrophy or ellipsoid disruption on SD-OCT was not always correlated with hypo-AF (Fig. 4c). Although FAF staging has been shown to correlate well with fundus photo in disease progression, this staging has limitations for predicting legal blindness in BCD patients. There are several reasons why FAF imaging may not be an appropriate tool for predicting legal blindness. First, because of the lutein pigments, the FAF signals in the foveola are usually lower in the central fovea than in other areas. Therefore, FAF signals in the foveal area cannot be used as an indicator of RPE activity in areas outside the fovea. Second, the RPE is not the only cell type that contributes to AF in the retina: inflammatory cells such as macrophages and microglia recruited by debris in the outer segment may also contribute to AF or hyper-AF [21, 22]. Third, FAF detects mainly RPE activities. While hypo-FAF accurately indicates RPE atrophy, it is still challenging to determine whether areas with preserved AF signaling retain normal RPE activity (Fig. 4c). Although quantitative FAF has been used to study RPE activity in certain retinal 
diseases [23, 24], there are limitations to the application of quantitative FAF in BCD because of its irregular distribution and patterns of RPE atrophy.

\section{Limitations}

There were several limitations to our study. First, the sample size was limited since BCD is not a common disease. Second, not all the patients in our study received genetic testing for CYP4V2 mutations. Third, there is no standard for classifying the amount of crystals and sclerotic vessels detected; thus, quantification depends on image modalities and may be subjective. In turn, future software analysis of standard images to quantify these features may provide more information associated with visual function. Fourth, this is a cross-sectional clinical study based on different patients in different stages of disease. Long term follow-up of these patients may further help us to understand the nature of the primary defect as well as structural or functional changes associated with this disease. Fifth, OCTA analysis or En face OCT images were not performed in our patient. Instead, we investigated multiple image modalities including Color fundus, FAF, NIR and OCT to assess this disease.

\section{Conclusions}

In conclusion, we found that NIR is a good diagnostic tool for BCD for detecting either crystalline deposits or sclerotic vessels. Furthermore, we suggest that central EZ disruption may be the best indicator for legal blindness as it can be assessed easily from SD-OCT images and correlates with BCVA. The resulting functional and structural correlation could help monitor disease progression in BCD and could be a good parameter for observing the effect of treatment in the future. Further study is required to identify ways of preventing the progression of this disease.

\section{Supplementary Information}

The online version contains supplementary material available at https://doi. org/10.1186/s12886-021-01811-y.

Additional file 1.

\section{Abbreviations}

BCD: Bietti crystalline dystrophy; BCVA: Best corrected visual acuity; NIR: Nearinfrared reflectance; FAF: Fundus autofluorescence; SD-OCT: Spectral domainoptical coherence tomography; EZ: Ellipsoid zone; CYP4V2: Cytochrome P450 family 4 subfamily $V$ member 2; RPE: Retinal pigment epithelium: OCTA: Optical coherence tomography angiography; ORT: Outer retinal tubulation; ROC: Receiver-operating characteristic; AUC: Area under the curve

\section{Acknowledgements}

The authors thank the retinal imaging technicians (Department of Ophthalmology, Chang Gung Memorial Hospital, Linkou \& Taipei) for technical assistance.

\section{Authors' contributions}

CYH, EYK, ALW, LKY, LL, WCW, CCL, KJC, and NKW contribute to the design of work. CYH, EYK, and ALW contribute to acquisition and analysis of the data. PKL, IWH, JR and KJC interpreted the patient data. CYH, KJC and NKW were a major contributor in writing the manuscript. All authors read and approved the final manuscript.

\section{Funding}

Publication of this article was supported in part by research grant of the Taiwan Ministry of Science and Technology MOST 106-2314-B-182A-041, the Chang Gung Memorial Hospital CMRPG3F1243, CMRPG3K1681. NKW is supported by National eye institute (US) NEI R01EY031354. The funders had no role in study design, data collection and analysis, decision to publish, or preparation of the manuscript.

\section{Availability of data and materials}

The dataset that used and analyzed during the current study are available from the corresponding author on reasonable request.

\section{Ethics approval and consent to participate}

The consents to all participate were not necessary because it was a retrospective chart review without any intervention, or identifiable pictures or data from the patients. This retrospective study (waiver of inform consents) was approved by the Institutional Review Board of Chang Gung Memorial Hospital, Taiwan (IRB 202001456B0). Four patients who received genetic tests had previously signed consent forms for another prospective study on "Genetic study in hereditary retinal and optic nerve diseases, (IRB 201601569B0(601)".

Consent for publication

Not applicable.

\section{Competing interests}

The author declared that they had no competing interests.

\section{Author details}

${ }^{1}$ Department of Ophthalmology, Chang Gung Memorial Hospital, Linkou Medical Center, Taoyuan, Taiwan. ${ }^{2}$ College of Medicine, Chang Gung University, Taoyuan, Taiwan. ${ }^{3}$ Department of Ophthalmology, Mackay Memorial Hospital, Hsinchu, Taiwan. ${ }^{4}$ Department of Ophthalmology, Edward S. Harkness Eye Institute, Columbia University Irving Medical Center, Columbia University, New York, USA. ${ }^{5}$ Department of Ophthalmology, Kaohsiung Medical University Hospital, Kaohsiung, Taiwan. ${ }^{6}$ Department of Ophthalmology, School of Medicine, Kaohsiung Medical University, Kaohsiung, Taiwan. ${ }^{7}$ Institute of Biochemical Science, National Sun Yat-Sen University, Kaohsiung, Taiwan. ${ }^{8}$ Department of Ophthalmology, Chang Gung Memorial Hospital, Keelung, Taiwan.

Received: 20 August 2020 Accepted: 11 January 2021

Published online: 04 February 2021

\section{References}

1. Li A, Jiao X, Munier FL, Schorderet DF, Yao W, Iwata F, et al. Bietti crystalline Corneoretinal dystrophy is caused by mutations in the novel gene CYP4V2. Am J Hum Genet. 2004;74(5):817-26.

2. Nakano M, Kelly EJ, Wiek C, Hanenberg H, Rettie AE. CYP4V2 in Bietti's crystalline dystrophy: ocular localization, metabolism of $\omega$-3-polyunsaturated fatty acids, and functional deficit of the p.h331p variant. Mol Pharmacol. 2012:82:679-86.

3. Bietti G. Ueber familiäres Vorkommen von "Retinitis punctata albescens" (verbunden mit "Dystro-phis marginalis cristallinea corneae"). Glitzern, des Glaskörpers und anderen degenerativen Augenver-änderungen. Klin Monatsbl Augenheilkd. 1937;99:737-56.

4. Bernauer W, Daicker B. Bietti's corneal-retinal dystrophy: a 16-year progression. Retina. 1992;12(1):18-20.

5. Mataftsi A, Zografos L, Milla E, Secrétan M, Munier FL. Bietti's crystalline corneoretinal dystrophy: a cross-sectional study. Retina. 2004;24:416-26.

6. Yuzawa M, Mae Y, Matsui M. Bietti's crystalline retinopathy. Ophthalmic Genet. 1986;7(1):9-12. 
7. Oishi A, Oishi M, Miyata M, Hirashima T, Hasegawa T, Numa S, et al. Multimodal imaging for differential diagnosis of Bietti crystalline dystrophy. Ophthalmol Retin. 2018;2:1071-7.

8. Li Q, Li Y, Zhang X, Xu Z, Zhu X, Ma K, et al. Utilization of fundus autofluorescence, spectral domain optical coherence tomography, and enhanced depth imaging in the characterization of Bietti crystalline dystrophy in different stages. Retina. 2015;35(10):2074-84.

9. Miyata M, Oishi A, Hasegawa T, Ishihara K, Oishi M, Ogino K, et al. Choriocapillaris flow deficit in Bietti crystalline dystrophy detected using optical coherence tomography angiography. Br J Ophthalmol. 2018;102: 1208-12.

10. Russell S, Bennett J, Wellman JA, Chung DC, Yu ZF, Tillman A, et al. Efficacy and safety of voretigene neparvovec (AAV2-hRPE65V2) in patients with RPE65-mediated inherited retinal dystrophy: a randomised, controlled, open-label, phase 3 trial. Lancet. 2017:390(10097):849-60

11. Varma R, Vajaranant TS, Burkemper B, Wu S, Torres M, Hsu C, et al. Visual impairment and blindness in adults in the United States. JAMA Ophthalmol. 2016;134(7):802-9.

12. Lin J, Nishiguchi KM, Nakamura M, et al. Recessive mutations in the CYP4V2 gene in east Asian and dystrophy. J Med Genet. 2005;42(6):e38.

13. Xiao X, Mai G, Li S, Guo X, Zhang Q. Identification of CYP4V2 mutation in 21 families and overview of mutation spectrum in Bietti crystalline corneoretinal dystrophy. Biochem Biophys Res Commun. 2011;409:181-6.

14. Hwang JC, Kim DY, Chou CL, Tsang SH. Fundus autofluorescence, optical coherence tomography, and electroretinogram findings in choroidal sclerosis. Retina. 2010;30(7):1095-103.

15. Harrison RJ, Acheson RR, Dean-Hart JC. Bietti's tapetoretinal degeneration with marginal corneal dystrophy (crystalline retinopathy): case report. Br J Ophthalmol. 1987;71(3):220-3.

16. Kaiser-Kupfer MI, Chan CC, Markello TC, Crawford MA, Caruso RC, Csaky KG, et al. Clinical biochemical and pathologic correlations in Bietti's crystalline dystrophy. Am J Ophthalmol. 1994;118(5):569-82.

17. Liu CF, Liu L, Lai CC, Chou JC, Yeh LK, Chen KJ, et al. Multimodal imaging including spectral-domain optical coherence tomography and confocal near-infrared reflectance for characterization of lacquer cracks in highly myopic eyes. Eye. 2014;28(12):1437-45.

18. Sampson DM, Alonso-Caneiro D, Chew AL, Lamey T, McLaren T, De Roach J et al. Enhanced visualization of subtle outer retinal pathology by en face optical coherence tomography and correlation with multi-modal imaging. PLoS One. 2016;11(12):e0168275.

19. Zweifel SA, Engelbert M, Laud K, Margolis R, Spaide RF, Freund KB. Outer retinal tubulation a novel optical coherence tomography finding. Arch Ophthalmol. 2009;127(12):1596-602.

20. Halford S, Liew G, Mackay DS, Sergouniotis PI, Holt R, Broadgate S, et al. Detailed phenotypic and genotypic characterization of bietti crystalline dystrophy. Ophthalmology. 2014;121:1174-84.

21. Wang NK, Fine HF, Chang S, Chou CL, Cella W, Tosi J, et al. Cellular origin of fundus autofluorescence in patients and mice with a defective NR2E3 gene. Br J Ophthalmol. 2009;93(9):1234-40.

22. Wang NK, Lai CC, Liu CH, Yeh LK, Chou CL, Kong J, et al. Origin of fundus hyperautofluorescent spots and their role in retinal degeneration in a mouse model of Goldmann-Favre syndrome. DMM Dis Model Mech. 2013: 6(5):1113-22.

23. Dysli C, Quellec G, Abegg M, Menke MN, Wolf-Schnurrbusch U, Kowal J, et al. Quantitative analysis of fluorescence lifetime measurements of the macula using the fluorescence lifetime imaging ophthalmoscope in healthy subjects. Investig Ophthalmol Vis Sci. 2014;55(4):2106-13.

24. Schachar IH, Zahid S, Comer GM, Stem M, Schachar AG, Saxe SJ, et al. Quantification of fundus autofluorescence to detect disease severity in nonexudative age-related macular degeneration. JAMA Ophthalmol. 2013; 131(8):1009-15.

\section{Publisher's Note}

Springer Nature remains neutral with regard to jurisdictional claims in published maps and institutional affiliations.

Ready to submit your research? Choose BMC and benefit from:

- fast, convenient online submission

- thorough peer review by experienced researchers in your field

- rapid publication on acceptance

- support for research data, including large and complex data types

- gold Open Access which fosters wider collaboration and increased citations

- maximum visibility for your research: over $100 \mathrm{M}$ website views per year

At BMC, research is always in progress.

Learn more biomedcentral.com/submissions 